\title{
Hábitos alimentarios y estado nutricional de niños y niñas que asisten a las Escuelas Deportivas Integrales del Instituto Nacional de Deporte, Región de Nuble
}

\author{
Eating habits and nutritional status of children attending the Integral Sports \\ Schools of the National Sports Institute, Nuble Region, Chile
}

\begin{abstract}
RESUMEN
La malnutrición por exceso se ha convertido en un problema de salud pública a través de todo el ciclo vital. El objetivo del estudio fue analizar los hábitos alimentarios y el estado nutricional de niños y niñas que asisten a las Escuelas Deportivas Integrales (EDI) del Instituto Nacional de Deporte en la comuna de Chillán. Se realizó evaluación antropométrica y aplicación de encuesta sobre hábitos alimentarios, que permitía medir alimentos consumidos de manera diaria, semanal y mensual de: frutas, verduras, lácteos, agua, carne, pescado, legumbres, golosinas dulces y saladas, productos fritos, comida rápida y embutidos. En los resultados, se confirma una alta prevalencia de malnutrición por exceso (58,7\%). Para el cumplimiento de las Guías Alimentarias Basadas en Alimentos (GABAS) para la población chilena, se observó sólo un $59,2 \%$ en frutas, $71,4 \%$ para verduras, $13,8 \%$ en pescado, $39,3 \%$ en legumbres y $66,3 \%$ en lácteos. Diariamente, más del $65 \%$ consume golosinas dulces y/o saladas. Semanalmente, la comida rápida y productos fritos se ingieren en un $66 \%$ y $79 \%$ respectivamente. Se sugiere la necesidad de realizar intervenciones multidisciplinarias de prevención y promoción de la salud, orientadas a mejorar estilos de vida saludables en la población infantil y así contribuir a disminuir los índices de morbilidad infantil.

Palabras clave: Estado nutricional; Ingesta alimentaria; Sobrepeso.
\end{abstract}

\footnotetext{
ABSTRACT

Over nutrition has become a public health problem over the life cycle. The objective of the study was to analyze the eating habits and nutritional status of children attending the Integral Sports Schools (ISS) of the National Sports Institute in Chillán, Chile. An anthropometric evaluation was performed; an eating habits survey, which measured the consumption of fruit, vegetables, dairy products, water, meat, fish, legumes, sweet and salty treats, fried products, junk food, and cold meats on a daily, weekly, and monthly basis was completed. Results confirmed a high prevalence of overnutrition (58.7\%). Complying with Food Based Dietary Guidelines for the Chilean Population, values were 59.2\% for fruit, $71.4 \%$ for vegetables, $13.8 \%$ for fish, $39.3 \%$ for
}

Pamela Castillo ${ }^{1}$, Jacqueline Araneda ${ }^{2 *}$, Anna Christina Pinheiro ${ }^{3}$.

1. Instituto Nacional de Deporte, Programa Crecer en Movimiento. Magíster en Salud Pública Universidad del Bío-Bío, Chillán, Chile. 2. Departamento de Nutrición y Salud Pública, Facultad de Ciencias de la Salud y los Alimentos, Universidad del Bío-Bío, Chillán, Chile. 3. Escuela de Nutrición y Dietética, Facultad de Medicina-Clínica Alemana, Universidad del Desarrollo, Santiago, Chile.

*Dirigir correspondencia a: Jacqueline Alejandra Araneda Flores. Departamento de Nutrición y Salud Pública. Facultad de Ciencias de la Salud y de los Alimentos. Universidad del Bío-Bío. Avenida Andrés Bello 720, Chillán. Chile. E-mail: jaraneda@ubiobio.cl

Este trabajo fue recibido el 06 de noviembre de 2019. Aceptado con modificaciones: 24 de enero de 2020. Aceptado para ser publicado: 08 de abril de 2020 .

legumes, and $66.3 \%$ for dairy products. More than $65 \%$ of the children ate sweet and/or salty treats on a daily basis. Weekly intake of junk food and fried products was $66 \%$ and $79 \%$, respectively. Multidisciplinary prevention and health promotion interventions are required to improve healthy lifestyles in children and thus contribute to reducing child morbidity rates.

Key words: Food intake; Nutritional status; Overnutrition.

\section{INTRODUCCIÓN}

La prevalencia de sobrepeso y obesidad ha aumentado en todo el mundo en las últimas décadas, convirtiéndose en un grave problema de salud pública en todo el ciclo vital, tanto a nivel internacional como nacional ${ }^{1,2}$. 
En Chile, los últimos datos de la Encuesta Nacional de Salud 2016-2017 (ENS 2016-2017), que consideró una muestra de hombres y mujeres de 15 y más años, muestran que la prevalencia del exceso de peso (sobrepeso, obesidad y obesidad mórbida) en el país, alcanza $74,2 \%{ }^{3}$. Por otra parte, en el último mapa nutricional desarrollado por la Junta Nacional de Auxilio Escolar y Becas (JUNAEB) el año 2018, se obtuvo cifras alarmantes de mal nutrición por exceso para pre-kínder (49\%), kínder (51\%), primero básico $(50 \%)$, quinto básico $(60 \%)$ y primero medio $(46 \%)^{4}$.

Según la Encuesta Nacional de Consumo Alimentario (ENCA), realizada en Chile los años 2010 y 2011, que tuvo por finalidad conocer los patrones de consumo, en el contexto de conductas y hábitos alimentarios de la población chilena, muestra que la prevalencia de sobrepeso y obesidad, en su conjunto, comprometen a casi la mitad de la población escolar de 6 a 18 años con un 46,6\%. En los niños de 6 a 13 años se obtuvo un $19,7 \%$ de sobrepeso y un $39,1 \%$ de obesidad, sin embargo, en las niñas con el mismo rango etario se alcanzó un $25,2 \%$ de sobrepeso y $23,6 \%$ de obesidad ${ }^{5}$.

Teniendo en consideración la naturaleza multifactorial de la mal nutrición por exceso, los expertos concuerdan en la importancia de reconocer y estudiar la valoración de la ingesta alimentaria en niños y adolescentes para la planificación y ejecución de intervenciones focalizadas e integradas, orientadas tanto a la prevención como al tratamiento de la enfermedad ${ }^{6,7}$.

La ENCA a través del Índice de Alimentación Saludable (IAS) arrojó que el 95\% de la población chilena no tiene una alimentación saludable y que debe realizar modificaciones en su alimentación. De la misma forma, se constató un bajo grado de cumplimiento de las recomendaciones sugeridas por las Guías Alimentarias Basadas en Alimentos (GABAS) (Cuadro 1) del Ministerio de Salud ${ }^{8}$, tanto para lácteos, frutas, verduras y pescados.

Por otra parte, ENS 2016-2017, que consideró sedentario a aquellos individuos que en los últimos 30 días no practicaron deporte o realizaron actividad física fuera de su horario de trabajo, durante 30 minutos o más cada vez, 3 o más veces por semana; muestra que el $86,7 \%$ de los chilenos es sedentario y de igual forma el $73,5 \%$ de los adolescentes de 15 a 19 años $^{3}$. Lo anterior, es consecuente con estudios nacionales realizados en este grupo etario, demostrando que los niños presentan un patrón de actividad predominantemente sedentario ${ }^{9,10}$.

Situación similar se observa según los datos proporcionados por el Sistema Nacional de Evaluación de Resultados de Aprendizaje (SIMCE) de actividad física del 2015, realizado en niños de $8^{\circ}$ año básico de los establecimientos escolares del país. De un total de 9.568 estudiantes, el $2 \%$ tiene un nivel satisfactorio en aspectos estructurales de la condición física (pruebas de abdominales cortos, salto largo a pies juntos, flexo-extensión de codos y flexión de tronco adelante), en relación a aspectos funcionales de la condición física (test de Cafra y de Navette.), sólo el 28\% logra un nivel satisfactorio ${ }^{11}$.

De la misma forma, un estudio realizado por investigadores internacionales y un grupo de universidades chilenas en materia de actividad física infantil, evidenció que en Chile, 7 de cada diez niños no cumple con los estándares de actividad física recomendado por la Organización Mundial de la Salud (OMS), la cual recomienda 180 minutos diarios de actividad física de cualquier intensidad para los pre-escolares y 60 minutos de actividad moderada o vigorosa para escolares y adolescentes, todos los días de la semana ${ }^{12}$.

Estudios demuestran que los niños y niñas con un estado nutricional normal, son los que dedican más tiempo a la práctica de actividad física en el colegio, mientras que los escolares con sobrepeso y obesidad disponen menor tiempo ${ }^{13,14}$. Por otra parte, se observó una disminución de la práctica de actividad física de los niños y niñas a medida que se incrementa la edad ${ }^{15}$.

El Gobierno de Chile consciente del problema del sedentarismo en los escolares, el año 2014 implementó el Programa Escuelas Deportivas Integrales (EDI) que tiene por finalidad proporcionar espacios de práctica de actividad física deportiva a niños y niñas entre 2 a 14 años de edad, el cual fue constituido por dos componentes (Escuelas y Encuentros) y cada uno de ello, está compuesto por cinco productos (jardín activo, escuelas de iniciación deportiva, escuelas de formación deportiva, escuelas deportivas de especialización y escuelas deportivas para niños y niñas en situación de discapacidad), los que están directamente relacionados con la edad de desarrollo, maduración y crecimiento de los participantes ${ }^{16}$.

En base a todo lo expuesto anteriormente, existe un gran consenso en la necesidad urgente de mejorar la alimentación y aumentar la actividad física que presentan los escolares. Es así, como las intervenciones a temprana edad como las desarrolladas por las EDI del Instituto Nacional de Deporte (IND), constituyen una de las muchas estrategias para enfrentar la obesidad en la población ${ }^{17}$.

Por consiguiente, el objetivo del presente estudio es analizar los hábitos alimentarios y el estado nutricional de niños y niñas que asisten a las EDI del IND en la comuna de Chillán. Se espera que esta investigación, sea un aporte al conocimiento científico existente, y que además puedan servir de insumos para el diseño de políticas públicas e intervenciones deportivas específicas, implementadas en la población infantil y adolescente.

\section{MATERIAL Y MÉTODO}

Se desarrolló un estudio analítico de corte transversal. El universo correspondió a 420 estudiantes que habitan en zonas urbanas de la comuna de Chillán, Región de Ñuble, y que participan en las EDI de básquetbol, fútbol, rugby y polideportivo (incluye distintas disciplinas o modalidades deportivas).

Mediante muestreo aleatorio simple se obtuvo una muestra de 196 niños y niñas. El tamaño muestral se calculó con un nivel de confianza del 95\% y aceptando un error máximo permisible del $5 \%$.

Dentro de los criterios de inclusión se consideró: que los niños y niñas hayan participado en las EDI durante al menos 
dos meses, presentar registros de medición antropométrica (peso, talla) y tener un rango de edad de 6 a 11 años (grupos de iniciación deportiva). Por otra parte, los criterios de exclusión fueron: presentar situación de discapacidad física y/o cognitiva, tener alguna enfermedad metabólica (diabetes mellitus, resistencia a la insulina, síndrome metabólico, dislipidemia e hipertensión arterial) o presentar trastornos de la conducta alimentaria. Toda la información fue obtenida a través de entrevistas con los niños y niñas y posteriormente corroborada con los registros de las fichas personales de cada participante de las EDI.

Para la caracterización socioeconómica, se utilizó una adaptación de la encuesta desarrollada por World Association of Market Research ${ }^{18}$ y utilizada por agencias de mercadeo en Chile, la que considera dos componentes: nivel de educación alcanzado por el principal sostenedor del hogar y categoría ocupacional del principal sostenedor del hogar, ambos consultados vía telefónica a los apoderados de los niños y niñas. Se construyeron categorías para nivel socioeconómico: bajo, medio (agrupando nivel medio, medio bajo y medio alto), alto y nivel educacional del apoderado: básica (básica incompleta o inferior, básica completa, media incompleta) Media (media completa, técnica incompleta, universitaria incompleta), superior o universitaria completa (universitaria completa, postgrado).

Además, fue registrado el tipo de disciplina deportiva practicada por el niño o niña en las EDIs.

El proyecto fue aprobado por el Comité de Bioética de la Universidad del Bío-Bío. Se solicitó la firma del consentimiento informado y asentimiento a los padres y/o cuidador de cada uno de los niños y niñas.

Hábitos alimentarios: Se determinó utilizando un cuestionario estructurado basado en las GABAS (Cuadro 1) y desarrollado especialmente para el estudio, que fue previamente validado por criterio de expertos en el ámbito alimentario nutricional. Este instrumento fue aplicado por método de entrevista a cada niño participante del programa EDI, por una nutricionista con experiencia en la obtención de datos alimentarios en niños y adolescentes. Dicha encuesta medía la cantidad de tiempos de comida realizados durante un día y categorías de alimentos consumidos de manera "diaria", "semanal", "mensual" y el "no consumo", incluyendo preguntas de alternativas, tales como: ¿Cada cuánto tiempo consume pescado o mariscos?, ¿Cada cuánto tiempo consume leguminosas (poroto, garbanzos, lentejas, otro)?, ¿Cuántas veces a la semana consume productos fritos (masas fritas, papas fritas y otros similares)?

Los grupos de alimentos observados fueron: frutas, verduras, lácteos, agua, carnes (vacuno, cerdo, pollo), pescados, legumbres, snacks dulces y salados, productos fritos, comida rápida, caracterizada por contener productos ultraprocesados y de alta densidad energética (hot-dog, hamburguesas, gaseosas), y embutidos (vienesas, jamón, longanizas).

Además, utilizando el mismo instrumento, se consultó sobre los alimentos que los niños/as consumían al desayuno y en la merienda.

\section{Estado nutricional}

Se calculó el Índice de Masa Corporal (IMC), previa medición de peso y talla, utilizando técnicas estandarizadas ${ }^{19}$. El peso se obtuvo mediante el uso de una balanza digital marca Seca, con una presión de 0,1 kg y la talla se medió, usando tallímentro marca Seca modelo 217, con una precisión de 0,1 milímetro. Luego se utilizó el Programa ANTHRO PLUS ${ }^{20}$ para la calificación del estado nutricional, y se categorizó según indicaciones del Ministerio de Salud (MINSAL) y OMS (OMS 2007); posteriormente se determinó el estado nutricional de los niños evaluados ${ }^{21}$. Todas las mediciones fueron realizadas por profesional nutricionista previamente entrenado y estandarizado.

\section{Análisis de la información}

Para las variables cuantitativas (edad, peso y talla), se calculó promedio y desviación estándar. Para las variables cualitativas (estado nutricional, nivel socioeconómico, disciplina y consumo de alimentos), se calcularon frecuencias absolutas y porcentajes con respecto al total y con sus respectivos intervalos de confianza (IC95\%). La relación de variables se realizó con

Cuadro 1. Guías alimentarias para la población chilena.

1. Para tener un peso saludable, come sano y realiza actividad física diariamente.

2. Pasa menos tiempo frente al computador o la tele y camina a paso rápido, mínimo 30 minutos al día.

3. Come alimentos con poca sal y saca el salero de la mesa.

4. Si quieres tener un peso saludable, evita el azúcar, dulces, bebidas y jugos azucarados.

5. Cuida tu corazón evitando las frituras y alimentos con grasas como cecinas y mayonesa

6. Come 5 veces verduras y frutas frescas de distintos colores, cada día.

7. Para fortalecer tus huesos, consume 3 veces en el día lácteos bajos en grasa y azúcar.

8. Para mantener sano tu corazón, come pescado al horno o a la plancha, 2 veces por semana.

9. Consume legumbres al menos dos veces por semana, sin mezclarlas con cecinas.

10. Para mantenerte hidratado, toma 6 a 8 vasos de agua al día.

11. Lee y compara las etiquetas de los alimentos y prefiere los que tengan menos grasas, azúcar y sal (sodio). 
la prueba de Chi-Cuadrado y Test exacto de Fisher, en el caso de frecuencias esperadas menores a 5. El procesamiento y análisis de los datos se realizó con el Software Estadístico Stata versión $11.0^{22}$, utilizando un nivel de significancia $\alpha=0,05$.

\section{RESULTADOS}

La muestra total fue de 196 niños de ambos sexos, de los cuales $14 \%$ correspondió al sexo femenino y $86 \%$ a masculino, con una media de edad de $9,2 \pm 1,6$ años. La tabla 1 muestra las características de los niños y niñas que participan de las EDI. En cuanto al nivel socioeconómico, más del $80 \%$ de los niños y niñas presentan un nivel socioeconómico medio. La disciplina mayormente practicada por los niños es el fútbol (70\%), mientras que en las mujeres es el básquetbol ( $52 \%$ ). En ambos sexos la actividad polideportiva alcanza proporciones cercanas al 20\%. En el estado nutricional, tanto en hombres como en mujeres predomina la

Tabla 1. Características de los niños y niñas que participan de las Escuelas Deportivas Integrales del Instituto Nacional del Deporte, según sexo.

\begin{tabular}{|c|c|c|c|c|c|c|}
\hline \multirow[t]{2}{*}{ Variables } & \multicolumn{5}{|c|}{ Sexo } & \multirow[b]{2}{*}{$\begin{array}{c}\text { Total } \\
\% \\
\text { (IC95\%) }\end{array}$} \\
\hline & $\mathbf{n}$ & $\begin{array}{c}\text { Masculino } \\
\% \\
\text { (IC95\%) }\end{array}$ & $\mathbf{n}$ & $\begin{array}{c}\text { Femenino } \\
\% \\
(\text { IC95\%) }\end{array}$ & $\mathbf{N}$ & \\
\hline \multicolumn{7}{|l|}{ Estado nutricional } \\
\hline Normal & 69 & $\begin{array}{c}40,8 \\
(33,6-8,5)\end{array}$ & 12 & $\begin{array}{c}44,4 \\
(26,8-63,5)\end{array}$ & 81 & $\begin{array}{c}41,3 \\
(34,5-48,4)\end{array}$ \\
\hline Sobrepeso & 49 & $\begin{array}{c}28,9 \\
(22,6-36,3)\end{array}$ & 6 & $\begin{array}{c}22,2 \\
(10,1-42,0)\end{array}$ & 55 & $\begin{array}{c}28,1 \\
(22,1-34,8)\end{array}$ \\
\hline Obesidad & 51 & $\begin{array}{c}30,2 \\
(23,7-37,6)\end{array}$ & 9 & $\begin{array}{c}33,3 \\
(18,0-53,1)\end{array}$ & 60 & $\begin{array}{c}30,6 \\
(24,5-37,4)\end{array}$ \\
\hline \multicolumn{7}{|c|}{ Nivel socioeconómico } \\
\hline Bajo & 14 & $\begin{array}{c}8,3 \\
(4,9-13,5)\end{array}$ & & - & 14 & $\begin{array}{c}7,1 \\
(4,2-11,7)\end{array}$ \\
\hline Medio & 146 & $\begin{array}{c}86,4 \\
(80,2-90,8)\end{array}$ & 19 & $\begin{array}{c}70,4 \\
(50,4-84,7)\end{array}$ & 165 & $\begin{array}{c}84,2 \\
(7,8-8,8)\end{array}$ \\
\hline Alto & 9 & $\begin{array}{c}5,3 \\
(2,7-9,9)\end{array}$ & 8 & $\begin{array}{c}29,6 \\
(15-49,5)\end{array}$ & 17 & $\begin{array}{c}8,7 \\
(5,4-13,5)\end{array}$ \\
\hline \multicolumn{7}{|l|}{ Disciplina } \\
\hline Básquetbol & 4 & $\begin{array}{c}2,4 \\
(0,8-6,2)\end{array}$ & 14 & $\begin{array}{c}51,9 \\
(33,1-70,0)\end{array}$ & 18 & $\begin{array}{c}9,2 \\
(5,8-14,1)\end{array}$ \\
\hline Fútbol & 119 & $\begin{array}{c}70,4 \\
(63,0-76,8)\end{array}$ & 3 & $\begin{array}{c}11,1 \\
(3,5-29,9)\end{array}$ & 122 & $\begin{array}{c}62,2 \\
(55,1-68,8)\end{array}$ \\
\hline Polideportivo & 35 & $\begin{array}{c}20,7 \\
(15,2-27,5)\end{array}$ & 6 & $\begin{array}{c}22,2 \\
(10,1-42,0)\end{array}$ & 41 & $\begin{array}{c}20,9 \\
(15,7-27,2)\end{array}$ \\
\hline \multirow[t]{2}{*}{ Rugby } & 11 & $\begin{array}{c}6,5 \\
(3,6-11,4)\end{array}$ & 4 & $\begin{array}{c}-14,8 \\
(5,5-34,0)\end{array}$ & 15 & $\begin{array}{c}7,7 \\
(4,6-12,3)\end{array}$ \\
\hline & & $x$ (D.E) & & $x(\mathrm{D} . \mathrm{E})$ & & $x($ D.E $)$ \\
\hline Edad (años) & & $9,30 \pm 1,65$ & & $9,29 \pm 1,69$ & & $9,29 \pm 1,65$ \\
\hline Peso $(\mathrm{Kg})$ & & $38,35 \pm 11,9$ & & $40,79 \pm 12,7$ & & $38,69 \pm 12,0$ \\
\hline Talla (cm) & & $136,8 \pm 11,7$ & & $139,0 \pm 11,8$ & & $137,1 \pm 11,7$ \\
\hline
\end{tabular}


malnutrición por exceso (sobrepeso y obesidad), con proporciones que alcanzan el $58 \%$ en hombres y $55 \%$ en mujeres. En el caso de sobrepeso, los hombres superan en 6 puntos porcentuales a las mujeres $y$ en obesidad la diferencia solo es de 3 puntos. Los resultados indican que el $40 \%$ de la muestra presenta un estado nutricional normal, independiente del sexo (44\% mujeres y $40 \%$ en hombres).

Con relación a los hábitos alimentarios, el 90\% de los niños y niñas señalan que consumen diariamente frutas, verduras y agua. También consumen a diario golosinas dulces y saladas en proporciones que superan el $65 \%$. Semanalmente, el $90 \%$ de niños y niñas señalan ingerir legumbres y 7 de cada 10 dice consumir pescado. Por otro lado, también señalan consumir embutidos, productos fritos y comida rápida, en proporciones de $81 \%, 79 \%$ y $68 \%$ respectivamente (Figura 1 ).
En cuanto a los alimentos consumidos en el desayuno, más del $50 \%$ de los niños y niñas, consumen cereales azucarados y leche y sus derivados, independiente de su estado nutricional. De igual forma, el $75 \%$ consumen pan o queque. Con relación a los agregados para el pan de tipo dulces, salados y lácteos, los niños y niñas señalan ingerirlos en proporciones de 28\%, 53\% y $34 \%$ respectivamente. En cuanto a la fruta, el 51\% de la muestra señalan no consumir estos alimentos al desayuno (Tabla 2).

En los alimentos consumidos en colaciones (Tabla 3), se observan diferencias estadísticamente significativas con el estado nutricional y no llevar colación $(p=0,017)$, llevar bebidas o jugos $(p=$ 0,042). En el límite de la significancia estadística se encuentra llevar pan $(p=0,055)$.

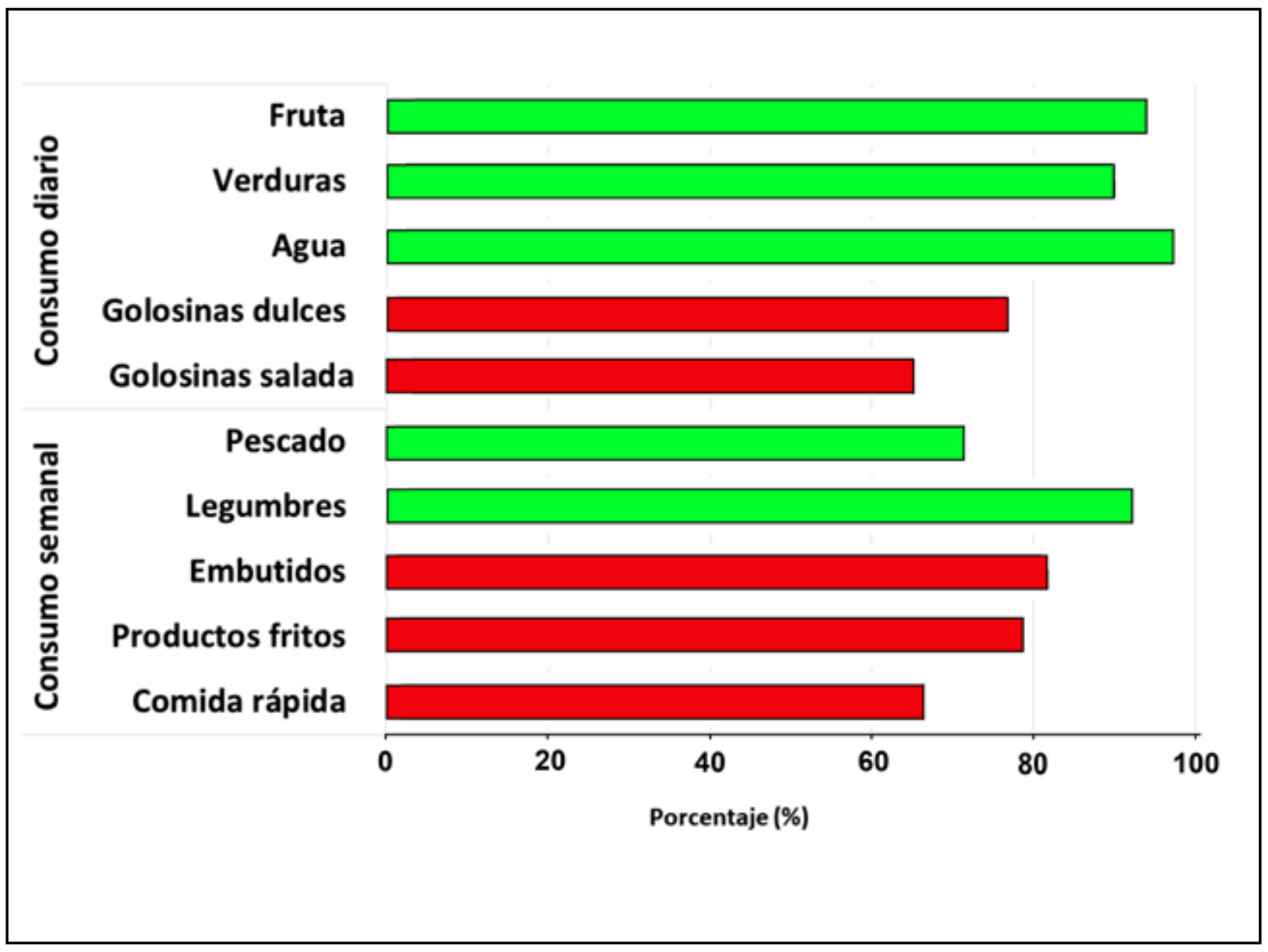

Figura 1: Porcentaje de alimentos consumidos diariamente y semanalmente por niños y niñas que participan de las Escuelas Deportivas Integrales del Instituto Nacional del Deporte $(\mathrm{n}=196)$. 
Tabla 2. Proporción de alimentos consumidos en el desayuno de niños/as que participan de las Escuelas Deportivas Integrales del Instituto Nacional del Deporte, según estado nutricional $(n=196)$.

\begin{tabular}{|c|c|c|c|c|c|c|}
\hline & & $\begin{array}{c}\text { Normal } \\
\text { n (\%) }\end{array}$ & $\begin{array}{c}\text { Estado nutricional } \\
\text { Sobrepeso } \\
\text { n (\%) }\end{array}$ & $\begin{array}{c}\text { Obesidad } \\
\text { n (\%) }\end{array}$ & $\begin{array}{l}\text { Total } \\
\text { n (\%) }\end{array}$ & $\mathbf{p}$ \\
\hline \multirow[t]{2}{*}{ Leche y derivados } & Consumo & $48(59,3)$ & $30(54,5)$ & $33(55,0)$ & $111(56,6)$ & 0,823 \\
\hline & No consumo & $33(40,7)$ & $25(45,4)$ & $27(45,0)$ & $85(43,4)$ & \\
\hline \multirow[t]{2}{*}{ Cereales azucarados } & Consumo & $46(56,8)$ & $24(43,6)$ & $33(55,0)$ & $103(52,5)$ & 0,289 \\
\hline & No consumo & $35(43,2)$ & $31(56,4)$ & $27(45,0)$ & $93(47,5)$ & \\
\hline \multirow[t]{2}{*}{ Pan, queque } & Consumo & $61(75,3)$ & $43(78,2)$ & $44(73,3)$ & $148(75,5)$ & 0,832 \\
\hline & No consumo & $20(24,7)$ & $12(21,8)$ & $16(26,7)$ & $48(24,5)$ & \\
\hline \multirow[t]{2}{*}{ Agregados dulces ${ }^{1}$} & Consumo & $26(32,1)$ & $12(21,8)$ & $17(28,3)$ & $55(28,1)$ & 0,424 \\
\hline & No consumo & $55(67,9)$ & $43(78.2)$ & $43(71,7)$ & $141(71,9)$ & \\
\hline \multirow[t]{2}{*}{ Agregados salados² } & Consumo & $45(55,6)$ & $30(54,5)$ & $29(48,3)$ & $104(53,1)$ & 0,674 \\
\hline & No consumo & $36(44,4)$ & $25(45,5)$ & $31(51,7)$ & $92(46,9)$ & \\
\hline \multirow[t]{2}{*}{ Agregado lácteo ${ }^{3}$} & Consumo & $26(32,1)$ & $18(32,7)$ & $23(38,3)$ & $67(34,2)$ & 0,716 \\
\hline & No consumo & $55(67,9)$ & $37(67,3)$ & $37(61,7)$ & $129(65,8)$ & \\
\hline \multirow[t]{2}{*}{ Fruta } & Consumo & $39(48,1)$ & $28(50,9)$ & $29(48,3)$ & $96(49,0)$ & 0,944 \\
\hline & No consumo & $42(51,9)$ & $27(49,1)$ & $31(51,7)$ & $100(51,0)$ & \\
\hline
\end{tabular}

${ }^{1}$ Mermelada, manjar, miel, otros; ${ }^{2}$ Mantequilla, huevo, cecinas, otros; ${ }^{3}$ Quesillo, queso, pasta de queso, otros.

Chi-cuadrado. Significancia estadística $\mathrm{p}<0,05$.

\section{DISCUSIÓN}

Los resultados de este estudio muestran una alta prevalencia de malnutrición por exceso, en donde 6 de cada 10 niños y niñas presenta sobrepeso u obesidad, a pesar de asistir regularmente a los espacios deportivos dispuestos por el IND, que consideran al menos 3 veces a la semana, 60 minutos de actividad física. Además, presentan patrones de consumo alimentario caracterizados por la ingesta de bebidas azucaradas y un bajo cumplimiento de las recomendaciones alimentarias diarias y semanales de frutas, verduras, pescados, legumbres y comida chatarra.

Los resultados del informe de la Organisation for
Economic Co-operation and Development (OECD) ${ }^{23}$, destacan a Chile como uno de los países con las mayores cifras de obesidad, lo que se puede atribuir a distintos factores, como los patrones alimentarios, sedentarismo y principalmente a la exposición de los niños y niñas a entornos que dificultan la adopción de estilos de vida más saludables. A pesar de lo anterior, Chile, es citado como referencia para diversas políticas públicas orientadas a enfrentar el problema, como por ejemplo el aumento del impuesto a las bebidas azucaradas (Ley 20.780) ${ }^{24} \mathrm{o}$ la implementación del mensaje frontal de advertencia a alimentos envasados altos en nutrientes críticos (Ley 20.606) ${ }^{25}$. 
Tabla 3. Proporción de hábitos alimentarios relacionados con las colaciones de niños/as que participan de las Escuelas Deportivas Integrales del Instituto Nacional del Deporte, según estado nutricional (n=196).

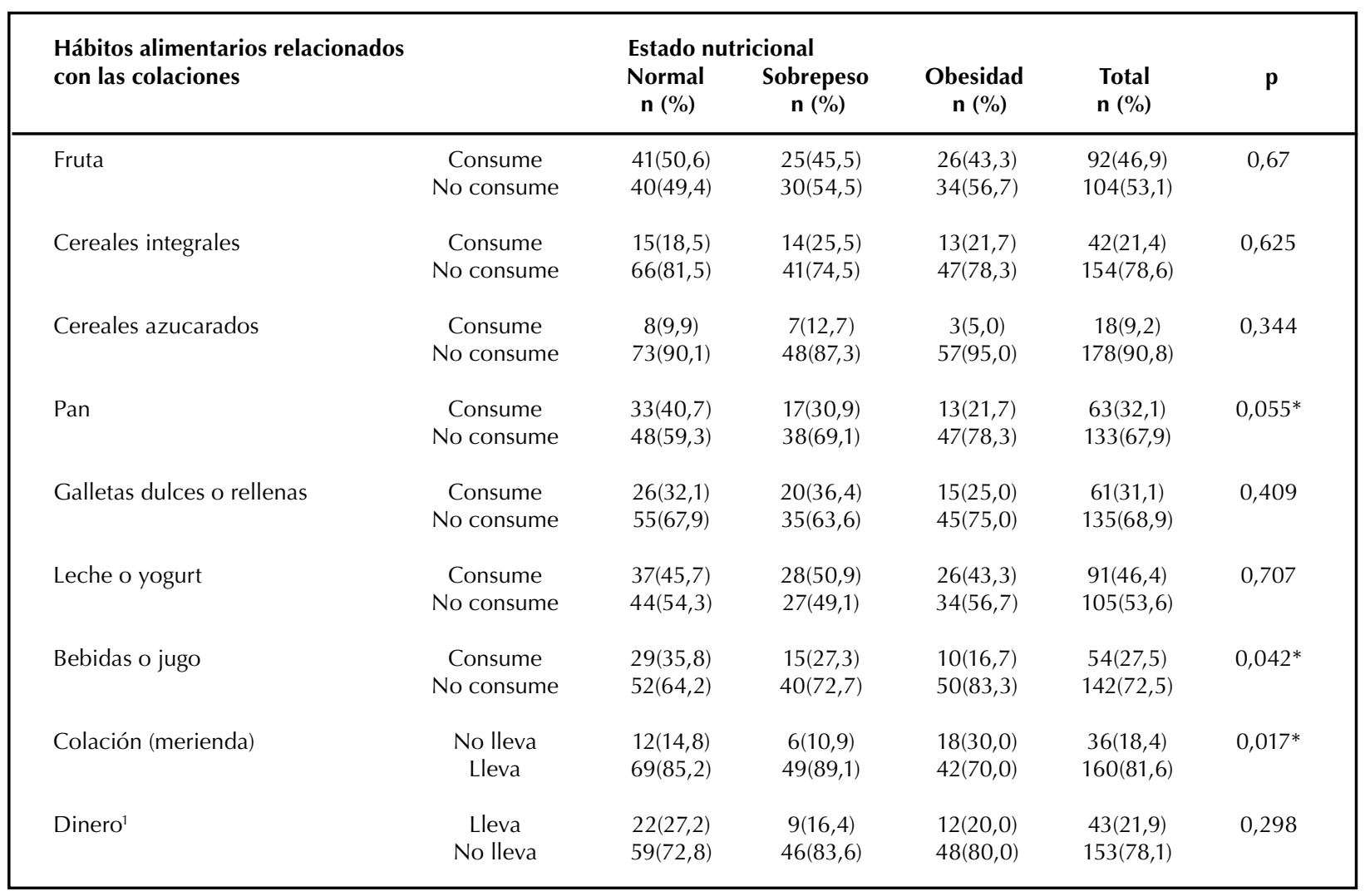

${ }^{1}$ Considera solo el dinero destinado para la compra de alimentos. Chi-cuadrado. Significancia estadística $\mathrm{p}<0,05$.

De los resultados obtenidos en el presente estudio, se destaca que, para el desayuno, los niños y niñas ingieren pan, con proporciones superiores al $70 \%$ y de preferencia con agregados salados como mantequilla, huevos y cecinas. Estos resultados son congruentes con lo reportado por la ENCA, que señala un alto consumo de pan, especialmente en los niveles socioeconómicos más bajos ${ }^{5}$, y con los resultados de la VIII Encuesta de Presupuestos Familiares, en donde refieren que el pan es un alimento con un alto gasto mensual en todas las zonas de Chile ${ }^{26}$. Lo anterior, dado que el pan es un alimento profundamente arraigado en la cultura chilena, y que por lo demás, tiene una alta densidad energética.

Por otra parte, las frutas y verduras solo son incluidas por la mitad de los sujetos de estudio en el desayuno y en las colocaciones, lo que nos permite pensar, que una parte de ellos no alcanza a cumplir la recomendación de las GABAS $N^{\circ} 6$ (8) que señala: "Come 5 veces verduras y frutas frescas de distintos colores, cada día". Estos resultados, son similares a los observados en otras poblaciones. Es así como en un estudio realizado en España, en el que se estudiaron a 562 niñas de niveles socioeconómicos medio, alto y bajo, de edades entre los 8 y 13 años, se encontró que solo $50 \%$ cumple con las recomendaciones de ingesta de este tipo de alimentos ${ }^{27}$.

En cuanto a proporción de alimentos consumidos en las colaciones de los niños y niñas que participan en las EDI, se observó que las bebidas y jugos presentaron diferencias estadísticamente significativas $(p=0,042)$, y un gradiente por estado nutricional, de forma que los obesos señalan consumirlas en menor proporción (16\%) que los con sobrepeso $(27 \%)$ y normalidad $(35 \%)$. El resultado parece contradictorio, considerando que un estudio realizado con una muestra representativa de escolares chilenos de 6 a 13 años, demostró que la ingesta de bebidas azucaradas se asocia con un mayor IMC, presentando un aumento de 0,13 del IMCz por cada porción de ingesta de $250 \mathrm{ml}$ ingerida ${ }^{28}$. No obstante lo anterior, hay que señalar que el diseño epidemiológico utilizado en la presente investigación es de corte transversal, en el que se pudo incluir a sujetos obesos que hubiesen comenzado tratamiento dietético recientemente, pero que aún se mantengan en esa condición, a pesar de haber cambiado sus hábitos alimenticios. 
Se encontró también que un alto porcentaje lleva dinero para la compra de alimentos en la escuela, lo que es concordante con otro estudio nacional realizado en 668 niños de 10 a 13 años, en el que reportan que el $60 \%$ de los estudiados disponía de dinero para comparar colaciones en la escuela ${ }^{29}$. En nuestro estudio, además se observó que los niños obesos señalaron llevar menos dinero para la compra de alimentos al compararlos con los con estado nutricional normal $(p=0,017)$.

La actividad física juega un papel importante en el desarrollo de los niños y niñas. Delgado et a ${ }^{30}$ han reportado una importante relación entre el nivel de actividad física y el estado nutricional, concluyendo que los escolares que realizan mayor número de horas de actividad física a la semana presentan mejor estado nutricional. Nuestro grupo de estudio, a pesar de ser parte de un programa estatal que está orientado a aumentar las horas de actividad física entre los escolares, presenta una elevada prevalencia de malnutrición por exceso, al igual que otras poblaciones de niños y niñas que no han realizado actividad física de manera regular y sistemática ${ }^{31,32}$.

La obesidad, es el resultado de complejas interacciones entre factores que están altamente interconectados como son los aspectos sociales, económicos, culturales, políticos, comerciales, entre otros ${ }^{33}$. Por esto, en los últimos años, ha cobrado mayor importancia la evaluación de los Ambientes Alimentarios que rodean a los individuos ${ }^{34}$ y la necesidad de reconocer a la obesidad como una pandemia, que en conjunto con la desnutrición y el cambio climático constituyen lo que se denomina la sindemia globa ${ }^{35}$. Enfrentar el problema requiere de acciones múltiples, dónde el aumento de la práctica constante de actividad física en todos los grupos de la población, es solamente una de las aristas.

En el caso del ambiente escolar, además de aumentar la oferta de actividad física para este grupo, es imprescindible avanzar en medidas estructurales que permitan una mayor oferta de alimentos saludables dentro y en las afueras de las escuelas, insertando de esta forma ambientes alimentarios más saludables, a través de intervenciones como la instalación de bebederos de agua, que faciliten el acceso al consumo de agua potable en forma digna, principalmente en escuelas que se encuentran en áreas vulnerables. Otra medida estructural imprescindible, es la inclusión de temas y actividades relacionadas a alimentación, nutrición y actividad física en el currículum escolar en forma transversal y homogénea, de tal forma que puedan estar contemplados en ejes de aprendizaje no tradicionales para esta temática, como es el caso de las asignaturas de Historia, Ciencias y Matemáticas.

Chile cuenta con políticas públicas orientadas al enfrentamiento de la obesidad y que son mencionadas como un ejemplo a seguir, tales como la Ley de Alimentos ${ }^{25}$, el Sistema Chile Crece Contigo ${ }^{36}$ el Sistema Elige Vivir Sano ${ }^{37}$, la Política Nacional de Alimentación y Nutrición ${ }^{38}$ entre otros. Es fundamental la integración de estas políticas, planes y programas a fin de lograr un objetivo común que es frenar el avance de la obesidad en el país y avanzar en la instalación de ambientes alimentarios y de actividad física más saludables para la población.

Una limitación de esta investigación, es que los datos fueron obtenidos en temporada de otoño invierno, época del año que se caracteriza por presentar temperaturas más bajas y frecuentes lluvias en la región en la cual la muestra fue obtenida, lo cual pudo haber influenciado en la frecuencia de asistencia a clases en las EDIs y nivel de actividad física de los niños y niñas evaluados. Otra limitante puede ser el sesgo de memoria ya que los sujetos debieron recordar la cantidad y frecuencia de distintos alimentos consumido por ellos al momento de responder la encuesta de hábitos alimentarios. También es necesario mencionar, la baja cantidad de mujeres estudiadas, lo que se debe principalmente a que la EDI están destinadas a disciplinas que son mayormente practicadas por niños, lo que hace imperativo incluir otras disciplinas que tengan mayor aceptación por las niñas.

A modo de conclusión, queda en evidencia que niños y niñas que realizan actividad física regular y sistemática, no tienen un mejor estado nutricional y hábitos alimentarios que la población general. Es imperativo apoyar medidas estructurales que permitan la generación de ambientes saludables tanto en el ámbito alimentario como de actividad física. Paralelamente, se sugiere la incorporación de programas de actividad física, que consideren la participación de la familia con un enfoque de género y con el apoyo de equipos multidisciplinarios de profesionales destinados promocionar estilos de vida saludables.

Agradecimientos: Al Instituto Nacional del Deporte, a todos los profesores de Educación física integrantes de esta institución y cada uno de los niños y niñas que participaron de esta investigación.

Las autoras declaran que no existe conflicto de interés.

\section{BIBLIOGRAFÍA}

1. Henríquez F, Lanuza F, Bustos L, González A, Hazbún J. Association between eating behavior and nutritional status in Chilean preschoolers. Nutr Hosp. 2018; 35: 1049-1053.

2. World Bank, UNICEF, WHO. Levels and trends in child malnutrition. Key findings of the 2018 edition [Consulted 2018, February 23]. Available from: http://www.who. int/nutgrowthdb/2018-jme-brochure.pdf?ua=1.

3. Ministry of Health Chile. National Health Survey 20162017 Chile. [Consulted 2018, February 23]. Available from: https://www.minsal.cl/wp-content/uploads/2017/11/ ENS-2016-17_PRIMEROS-RESULTADOS.pdf

4. National Board of School Aid and Scholarships (JUNAEB). Chile. Nutritional map report [Consulted 2019, abril 12]. Available from: https://www.junaeb.cl/wp-content/ uploads/2013/03/Mapa-Nutricional-2019-1.pdf

5. Ministry of Health Chile. National Survey on Food and Nutritional Consumption (ENCA), Final Report 2014 [Consulted 2018, February 23]. Available from: https:// www.minsal.cl/sites/default/files/ENCA-INFORME_FINAL. $p d f$. 
6. Castillo V, Escalona J, Rodríguez C. Food habits in chilean schoolchildren. Comparative analysis by type of educational establishment. Rev Chil Nutr. 2016; 43: 6-11.

7. Rodrigo C, Escauriaza B, Aranceta J, Allúe I. Dietary assessment in children and adolescents: issues and recommendations. Rev Esp Nutr. 2015; 21: 72-80.

8. Ministry of Health Chile. Food Based Dietary Guidelines. Revision and update. Final report. 2013. Santiago (CL): Ministry of Health; 2014.1-141 Available from: https://www.minsal.cl/portal/url/item/ dde0bc471a56a001e040010165012224.pdf

9. Herrera C, Lirab M, KainJ. Socioeconomic vulnerability and obesity in Chilean schoolchildren attending first grade: comparison between 2009 and 2013. Rev Chil Pediatr. 2017; 88: 736-743

10. Álvarez C, Ramírez $R$, Martínez C, Vallejos A, Jaramillo J, Salas $C$, et al. Hypertension in relation to nutritional status, physical activity and ethnicity in Chilean children aged 6 to 13. Nutr Hosp. 2016; 33: 220-225.

11. Education Quality Assurance Agency. National Study on Physical Education Report 8th grade school, 2015. Santiago (CL): Education Quality Assurance Agency;1-64. Available from: http://archivos.agenciaeducacion.cl/ Informe_Nacional_EducacionFisica2015.pdf

12. Aguilar N, Cortínez A, Leppe, Balboa T, Cobos C, Lemus $N$, et al. Chile's report card on physical activity: contributing to a global initiative for more active children and adolescents. Rev Med Chile. 2016; 144: 267-268.

13. Godoy A, Valdés $P$, Fariña C, Cárcamo $F$, Medina B, Meneses E, Gedda R, Durán S. Association between fitness, nutritional status and academic performance in physical education students. Nutr Hosp. 2015; 32: 1722-1728.

14. Godard C, Román M, Rodríguez M, Leyton B, Salazar G. Variability of physical activity in 4 to 10-year-old children: a study by accelerometry. Arch Argent Pediatr. 2012; 110: 388-393.

15. Valdés Pablo, Godoy A, Herrera T, Álvarez M, Durán $S$. Association between nutritional status and phisical activity at school in chilean children 4 to 14 years. Nutr Clín Diet Hosp. 2014; 34: 57-63.

16. Ministry of Sport, Chile. Integral Sports Schools [Internet]. Santiago (CL): Ministry of Sport 2018 [updated 2019 february 23; cited 2019 september 23]. Available from: https://www.ind.cl/deporte-formativo/

17. Menor M, Aguilar M, Mur N, Santana C. Effectiveness of health care educational interventions. A systematic review. Medisur. 2017; 15: 71-84.

18. Adimark, The Socio-Economic Level Esomar, 2000. Santiago (CL): Admark;1-8. Available from: https:// www.microweb.cl/idm/documentos/ESOMAR.pdf

19. Salvador G, Palma L, Puchal A, Vilá $M$, Miserachs $M$, Illan M. Dietetic interview. Tools for data collection. Rev Med Univ Navarra. 2016; 50; 46-55.

20. World Health Organization. WHO Multicentre Growth Reference Study Group. WHO Child Growth Standards: Length/height-for-age, weight-for-age, weight-forlength, weight-for-height and body mass index-for-age: Methods and development. Geneva (SW): World Health Organization; 1-77. Available from: https://www.who. int/childgrowth/standards/Technical_report.pdf?ua=1

21. Ministry of Health, Chile. Standards for the Nutritional
Assessment of Boys, Girls and Adolescents from 5 to 19 years old, 2016. Santiago (CL): Ministry of Health;1-76 Available from: https://www.previenesalud.cl/assets/ PDF/normas/2016-norma-evaluacion-nutricional.pdf

22. Stata Corp LLC. Stata 16: Software for Statistics and Data Science [Internet]. Stata Corp LLC; 2019 [updated 2019 March 20; cited 2019 september 23]. Available from: https://www.stata.com/

23. OECD. The Heavy Burden of Obesity: The Economics of Prevention, 2019. Paris (FR): OECD Publishing;16-238. Available from: https://www.oecd.org/health/the-heavyburden-of-obesity-67450d67-en.htm

24. Congress National Library [Internet]. Valparaiso (CL): National Congress;2014. Law 20.780. Tax reform that modifies the income tax system and introduces various adjustments to the tax system;2020 [cited 2019 Sep 20]. Available from: http://bcn.cl/1nfzd

25. Congress National Library [Internet]. Valparaiso (CL): National Congress;2013. Law 20.606. On nutritional composition of food and its advertising; 2013 [cited 2019 Sep 20]. Available in: http://www.leychile.cl/ Navegar?idNorma $=1041570$

26. National Statistical Institute. VIII Survey on Family Budgets (EPF). Main results report. Santiago (CL): National Statistical Institute;21-27. Avalilable from: https://www.ine.cl/estadisticas/sociales/ingresos-ygastos/encuesta-de-presupuestos-familiares

27. Rosa A, García E, Rodríguez Pedro, Pérez J, Tárraga M, Tárraga P. Physical activity, physical fitness and quality of diet in schoolchildren from 8 to 12 years. Nutr Hosp. 2017; 34: 1292-1298.

28. Araneda I, Bustos P, Cerecera F, Amigo H. Intake of sugar-sweetened non-alcoholic beverages and body mass index: A national sample of Chilean school children. Salud Pública Méx. 2015; 57: 128-134.

29. Bustos Z Nelly, Kain B Juliana, Leyton D Bárbara, Olivares C Sonia, Vio del R Fernando. Snacks usually consumed by children from public schools: motivations for their selection. Rev Chil Nutr. 2010; 37: 178-183.

30. Delgado P, Caamaño F, Osorio A, Jerez D, Fuentes J, Levin $E$ et al. Body image and self-esteem in children according to their nutritional status and frequency of physical activity. Rev Chil Nutr. 2017; 44: 12-18.

31. Vio del R F, Lera L, González C, Fierro M, Salinas J. Food and nutrition diagnosis of third to fifth grade school-age children from the La Reina neighborhood, Santiago de Chile. Rev Chil Nutr. 2017; 44: 244-250.

32. Muros Muros J, Cofre B, Zurita F, Castro M, Linares $M$, Chacon R. Relationship between physical fitness, physical activity, and different anthropometric parameters in school children in Santiago (Chile). Nutr Hosp. 2016; 33: 314-318.

33. Huamaní C, Rey de Castro J. Drowsiness and sleep characteristics in schoolchildren in an urban district of Lima, Peru. Arch Argent Pediatr. 2014; 112: 239-241.

34. Rioseco R, Egaña D, Galvez P, Masferrer D. Conceptual framework on the conditioning factors of food environments in Chile. Santiago (CL); Ministry of Health; 2016. 1-84. Available from: http://www. bibliotecaminsal. $\mathrm{cl} /$ marco-conceptual-sobre-los-factores-condicionantesde-los-ambientes-alimentarios-en-chile/

35. Swinburn BA, Kraak VI, Allender S, Atkins VI, Baker PI, Bogard JR, et al. The Global Syndemic of Obesity, 
Undernutrition, and Climate Change: The Lancet Commission report. Lancet. 2019; 393: 10173.

36. Congress National Library [Internet]. Valparaiso (CL): National Congress;2009. Law 20.379. Creates the Intersectoral Social Protection System and Institutionalizes the Subsystem for Comprehensive Protection of Children "Chile Grows with You"; 2019 [cited 2019 Sep 20]. Available from: http:// www.leychile.cl/Navegar?idNorma $=1006044 \& r=2$

37. Congress National Library [Internet]. Valparaiso (CL):
National Congress;2013. Law 20.670. Creates the System Choose to Live Health; 2019 [cited 2019 Sep 20]. Available from: http://www.leychile.cl/Navegar?idNorma=1051410

38. Zamora P, Rodriguez L, Pinheiro A, Pizarro T. National Food and Nutrition Policy. Santiago (CL); Ministry of Health; 2017.1-81. Available from: http://www. bibliotecaminsal.cl/wp/wp-content/uploads/2018/01/ BVS_POL\%C3\%8DTICA-DE-ALIMENTACI\%C3\%93N$Y-N U T T R I C I \% C 3 \% 93 N . p d f$ 